\title{
Wearable systems for e-health and wellbeing
}

\author{
Guglielmo Cola $^{1} \cdot$ Alessio Vechio $^{1}$
}

Published online: 4 July 2017

(C) Springer-Verlag London Ltd. 2017

Wearable devices, such as smartwatches and fitness bands, are becoming a key element of our lives. They are used in an always increasing number of activities, for example during sport sessions for keeping track of energy expenditure, or when walking as unobtrusive pedestrian navigation systems. In general, these devices are worn continuously throughout the day and thus provide the opportunity to gather information about their users with unprecedented levels. In addition, many wearable devices are directly worn over the skin and they may include sensors not available on common smartphones (e.g., for monitoring the user's heart rate). As a consequence, they are particularly suitable for those medical applications where continuous monitoring is fundamental. At the same time, the massive amount of information collected through these devices is enabling novel applications in the context of ehealth and wellbeing. Finally, it is known that abundance of information promotes an effective management of patients' condition, and a well-informed patient is more likely to conduct a healthy lifestyle.

This issue of Personal and Ubiquitous Computing collects recent, original research in the area of wearable systems for ehealth and wellbeing. Fourteen papers were initially submitted

Alessio Vecchio

alessio.vecchio@unipi.it

Guglielmo Cola

guglielmo.cola@iet.unipi.it

1 Dipartimento di Ingegneria dell'Informazione, University of Pisa, Pisa, Italy to this issue. After two rounds of review, four of them were finally accepted for publication.

The first paper, "Sleep behavior assessment via smartwatch and stigmergic receptive fields", presents a method for automatic assessment of sleep quality using a smartwatch. Heartbeat rate and wrist motion samples are processed using computational stigmergy, a bio-inspired technique that relies on digital pheromone marks.

The second paper, "Social Recommendations for Personalized Fitness Assistance", presents a novel framework-PRO-Fit-aimed at engaging users in fitness activities. The proposed framework minimizes the need for user input and proactively generates personalized fitness schedules. Collaborative filtering and social network information are exploited to automatically provide activities and fitness buddy recommendations.

The third paper, "Robust Orientation Estimate via Inertial Guided Visual Sample Consensus", presents a method for estimating the orientation of body joints using a wearable camera paired with an Inertial Measurement Unit (IMU). Visual information is used to correct the drift of the IMU, whereas information produced by the IMU enables more accurate and efficient image-based estimation.

The last paper, "SVM-based classification method to identify alcohol consumption using ECG and PPG monitoring", presents a method for detecting alcohol intoxication that is compatible with the requirements of wearable devices. Cardiac activity, observed through simple sensor configurations, is given as input to a classification system in charge of estimating the status of the user.

Finally, as co-guest editors of this issue, we would like to thank all the authors for their contributions. 